\title{
Desigualdad de género $y$ políticas públicas en áreas naturales protegidas
}

Gender inequality and public policies in protected areas.

Martha Cecilia Jaramillo Cardona Doctora en Ciencias Sociales co Colegio de la Frontera Norte, México) y Maestría en Desarrollo Regional de la Pontificia Universidad Javeriana (Bogotá). Docente e investigadora de tiempo completo de la Facultad de Economía y Relaciones Internacionales de la Universidad Autónoma de Baja California (UABC), sede Tijuan. Encargada de la Academia de Ciencia Sociales y Humanas de la Facultad de Nivel 1 del Sistema Nacional de Nivel 1 del Sisterna mcjaramu06@hotmail.edu.m.

Salvador Adame Góme Doctor en Ciencias

especialidad en estudios regionales ( Colegio de la Frontera Norte, México) con Maestría en Desarrollo Turistico co especialidad en planeación (Universida Investigador de Tiempo Compente Universidad Estatal de Sonora, sede S Luis Rio Colorado.

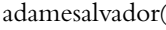

Fecha de recepción:

1.5.2017

Fecha de aprobación: 26.7.2017

\section{Resumen}

Este trabajo discute la forma en que se visualiza la desigualdad de género en los procesos de desarrollo en el Ejido Luis Encinas Johnson localizado dentro del municipio de San Luis Río Colorado Sonora, en la parte sureste del Valle Agrícola de Mexicali, área natural protegida caracterizada por políticas públicas en contradicción, una de conservación y otra de desarrollo humano. Se reflexiona sobre los impactos sociales, económicos y ambientales que la zona de estudio ha presentado como consecuencia de su declaración como área natural protegida, reconocimiento que vino a transformar la visión de las políticas públicas de conservación y el papel que deben cumplir los ejidatarios que viven en ella, especialmente las mujeres cabeza de familia, que se sienten excluidas por los programas públicos que allí se desarrollan por su condición de mujeres.

Palabras clave: desigualdad de género - políticas públicas - áreas naturales protegidas - conservación - desarrollo humano - México.

Abstract

This paper discusses gender inequality observed within the development process of the Ejido Luis Encinas Johnson, located at the Municipality of San Luis Rio Colorado, Sonora, in the Southeast region of the Agricultural Valley of Mexicali. The Ejido was declared a 
protected area on which the government established a set of conflicting public policies, one policy for conservation and a second policy for human development. Our aim is to reflect upon the social, economic and environmental impacts of this zone after it was designated a protected area. This act changed the way conservation public policies are envisioned and the role that the population must adopt in order to comply with the new regulations. This was especially challenging for women who are head of their families, for they feel excluded from public programs because of their gender.

Key-words: gender inequality - public policies - protected areas- conservation - human development- Mexico

\section{Introducción}

Existe una amplia discusión teórica sobre el término desigualdad, desde construir su definición a partir de distintas aristas del conocimiento, hasta cuestionar su concepción, práctica y uso en diversos órdenes del comportamiento humano, donde la claridad y opacidad de la palabra por sí misma es discutida. Debatir la desigualdad nos lleva a considerar las propuestas teóricas de justicia de Rawls (1997) y de libertad de Sen (1993) quienes de manera específica identifican que la desigualdad no se representa necesariamente por la falta de bienes; para Rawls consiste en el respeto por uno mismo, mientras que para Sen lo relevante son las aptitudes básicas. Ambas propuestas han permitido la teorización sobre la justicia en las ciencias sociales.

En términos de Caballero (2006) tanto la justicia como la libertad constituyen un acuerdo personal racional y una situación igualitaria que tiene validez universal e incondicional. Por lo tanto, pensar en igualdad conlleva necesariamente al manejo de valores en función de la imparcialidad, bajo la condición que ésta nos conduce irremediablemente a resultados imparciales. Comprendida de esta manera la justicia, entonces el efecto razonable mejora la influencia de factores naturales y sociales que aseguran el tratamiento equitativo de las distintas concepciones del bien.

Esta discusión referida a la justicia y la libertad permite trabajar la desigualdad como una consecuencia de la injusticia social y económica, las cuales se miden tradicionalmente utilizando el consumo, el ingreso u otros indicadores de riqueza (PNUD, 2013). Esta de- rivación nos indica que la libertad no es otra cosa más que el derecho igual de todas las personas a un extenso cúmulo de libertades, pero también se reconoce que las desigualdades económicas y sociales se determinan con la acción ventajosa de unos individuos sobre otros, quienes son sometidos de manera directa e indirecta. Estas nociones de justicia de Rawls, muestran una idea clara de cómo se construye el principio de diferencia

Buscando explicar este principio para entender la desigualdad, se atiende la propuesta de Ferrajoli y Carbonell (2005), quienes explican que la igualdad y la diferencia no son antónimas, por el contrario se exigen mutuamente, dado que la igualdad no implica homogeneidad en relación con las formas de ser, actuar y pensar de las personas. Esta relación analizada desde el ámbito del derecho determina que las diferencias no se valorizan ni se desvalorizan, no se tutelan ni se reprimen, no se protegen ni se violan estrictamente se les ignora. Pero se asume que la jerarquía de identidades sí se obtiene por acciones valorizadas por razón de sexo, nacimiento, etnia, fe religiosa, lengua, renta, entre otras (Lamas, 1986)

Entonces, es un hecho que las diferencias existen entre las personas, ya sea de índole natural, social o cultural, por lo tanto, distinguen e individualizan a los sujetos expresando su identidad y originalidad, características que deben ser valoradas y protegidas por el derecho (Ruiz, 2009). Estas desigualdades, ya sean económicas, sociales o políticas son discrepancias que existen tanto en el goce como en el ejercicio de los derechos de los individuos, y en tanto que profesen niveles legales diversos se traducen en desventajas para unos y ventajas para otros, modelos que deben desaparecer con base en el derecho, no sólo escrito, sino en palabra.

Actualmente, como una característica de nuestra sociedad a pesar de su avance en el combate a la desigualdad de género, se manifiesta abiertamente en la ocultación de ésta en el lenguaje, sobre todo cuando entendemos que la palabra es el elemento más influyente en la formación del pensamiento de una sociedad. Esto nos conduce a escudriñar en la opinión de Pateman, citada por Postigo (2006), cuando menciona que el pensamiento político establecido ha evidenciado la obediencia femenina como una consecuencia de la debilidad fisica e intelectual de las mujeres, y como resultado de un acto de consentimiento, aunque las mujeres no lo hayan negociado. Bajo esta lógica la mujer fue incorporada a una esfera que es y no civil el ámbito privado, es parte de la sociedad pero separado de la esfera civil (Postigo, 2006).

En este sentido, y apoyados en el argumento de la modernidad, las diferencias biológicas y psíquicas inscritas a los sexos han constituido la base que justifica la división de la ciudadanía en dos esferas: 1) la esfera civil y 2) la esfera doméstica. La primera, en palabras de Mejías y Henríquez (2012), se manifiesta por la conquista de espacios y poder político, una lucha que aboga por una ampliación de derechos humanos y ciudadanos y una 
real participación democrática en ambos sexos; mientras que la segunda ha limitado el acceso de las mujeres a la esfera pública en donde se desarrolló la sociedad civil (Varela, 2012). Esta desigualdad explica, según Bourdieu (2007), la eficiencia masculina en un modelo de legitimación al inscribir lo femenino en una construcción social biologizada.

A lo largo de la historia de las sociedades, los estudios sobre desigualdad de género han sido abordados desde dos ámbitos de acción: público y privado (Soto, 2013; Postigo, 2006) En ellos se pone en evidencia cómo desde siempre la mujer ha sido erróneamente considerada un objeto y no un sujeto de derecho, subordinada y discriminada a la imagen del hombre dominante como jefe y proveedor de la familia, limitándola culturalmente a la simple figura del hogar (Soto, 2013), situación que ha cambiado paulatinamente con los espacios ganados por las mujeres en diversos terrenos de la vida laboral, política y social.

No obstante este avance significativo en la participación de la mujer en el escenario económico, político y social muestra a través de una amplia bibliografia que las desigualdades de género, al igual que otras formas de inequidades, exhiben otros aspectos simbólicos y estructurales, ideológicos y materiales, interactivos e institucionales que se articulan para producir tal efecto (Oliveira, 2007; Maffia, 2006). Este conjunto de normas, valores y prácticas que establecen relaciones jerárquicas entre hombres y mujere dentro y fuera del grupo social o familiar, permite explicar el nivel de restricción de la mujer al acceso en actividades, funciones y decisiones en su vida y comunidad.

Aunque este fenómeno de relaciones jerárquicas entre género se ha extendido, se agudiza aún más cuando se presenta en el ámbito rural. A pesar de todos los avances en materia de igualdad social, la realidad nos exhibe que a pesar de estar en pleno siglo XXI sigue prevaleciendo lo urbano sobre lo rural (Warman, 2001), por consecuencia, los pocos estudios sobre género en áreas naturales protegidas (en adelante ANP) demuestran que gran parte de las mujeres que habitan estas zonas o dependen de ellas, no tienen acceso ni control sobre estos recursos (Lara y Vizcarra, 2008).

Esta realidad de desigualdad de género en ANP, se sustenta en la percepción que tienen las mujeres respecto a los hombres en las actividades productivas, donde es común que ésta se construya en función del uso específico de los recursos según el sexo (Velázquez, 2005). Entonces, el gran reto de las políticas públicas estriba, por tanto, en asegurar la equidad entre los géneros, tomando en cuenta que las relaciones de poder se reproducen jerárquica y asimétricamente entre hombres y mujeres, donde ellas son las más afectadas en cuanto al acceso, manejo y control de los recursos naturales.

El desarrollo de este trabajo pretende desde la metodología cualitativa, a través de cinco entrevistas en profundidad a mujeres ejidatarias y servidores públicos, realizar un análisis crítico y reflexivo de lo que significa el diseño de las políticas públicas en las ANP, al mismo tiempo de examinar la forma en que estas abordan el tema de la exclusión de las mujeres que habitan el ejido, por el hecho de ser "mujeres", sobre todo cuando son cabeza de familia.

\section{Material y Método}

Para este artículo se empleó información procedente de entrevistas en profundidad a servidores públicos responsables de diseñar las políticas públicas ambientales y a mujeres habitantes del Ejido Luis Encinas Johnson localizado en el municipio de San Luis Río Colorado (estado de Sonora), en la porción sureste del Valle Agrícola de Mexicali que hace parte de la "Reserva de la Biosfera Alto Golfo de California y Delta del Río Colorado". ${ }^{1}$ El objetivo de la investigación consistió en identificar, desde la visión institucional y la de las ejidatarias, la forma en que se deciden y llevan a cabo los programas de conservación ambiental para esta zona sin tener en cuenta una visión de género. ${ }^{2}$

\section{Políticas públicas y áreas naturales protegidas: un escenario} de contradicción y desigualdad social

Uno de los asuntos que mayor debate político, intelectual y académico ha suscitado en diversos ámbitos de la vida pública mexicana en los últimos años, es el relacionado con el diseño, implementación y evaluación de las políticas públicas de bienestar. Desde siempre, ha sido una constante que las políticas de prosperidad se construyan desde capacidades de atención a exigencias con características homogéneas y al mismo tiempo desiguales, analizadas y gestionadas con carácter estricto y administrativo (Subirats y Gomá, 2000).

En este entramado de desigualdad, la hechura de las políticas públicas en México no se efectúa a través de algún dispositivo de observación de los hechos, sino que el hacedor de las políticas trabaja con supuestos y categorías que configuran la realidad, motivo por el cual opera con esquemas, modelos y marcos de referencia implícitos y explícitos (Aguilar, 2007). Esta forma de actuación en la estructura histórica, política, económica y social de hacer políticas permea de manera directa e indirecta su proceso de elaboración, de tal forma que se siguen tomando las decisiones en los nichos de poder, excluyendo las demandas de las comunidades y población en general (Viola, 2000).

Por supuesto, no es extraño encontrar que esta manera de concebir políticas públicas se evidencia claramente en las ANP, espacios geográficos que manifiestan costos de oportunidad (CONANP, 2009), aunque sobre esto existan opiniones encontradas (PNUMA, 2007). El discurso ambiental para las ANP se muestra en dos vertientes, por un lado se menciona que las reglas de operación marcadas en los Planes de Conservación y Manejo promueven un mayor bienestar para la población que en ellas reside; por otro, se argumenta que las condiciones de pobreza en que se desarrollan estas zonas protegidas tienen un efecto negativo en los objetivos de las mismas, generado por la insuficiencia 
en financiamiento y personal para atender la gran dimensión del área. Esta circunstancia se puede encontrar en los Programas de Empleo Temporal (PET) y el Programa de Desarrollo Regional Sustentable (PRODERS), que destacan la escasa inversión en el desarrollo social que implementan las ANP, pero sobre todo, evidencian que la perspectiva de género no está definida ni mucho menos incorporada como algo relevante.

La ausencia de la perspectiva de género y sus impactos en la desigualdad social en el funcionamiento efectivo de las ANP en el mundo, puede corroborase en la amplia literatura que se ha producido al respecto, la cual resume que esta condición de desigualdad de género no solo puede detonar las condiciones de pobreza, sino que la restricción de acceso de la mujer en los programas de conservación y manejo de los recursos naturales, tiende a reducir las oportunidades de mejora del desarrollo humano en las comunidades locales (Riemann, Santes-Álvarez y Pombo, 2011). Sin embargo, a pesar del incremento de estudios sobre las ANP, es un hecho que existe poca información documentada respecto de las repercusiones sociales, económicas, ambientales y culturales que las prohibiciones en el aprovechamiento cotidiano de los recursos naturales y culturales aplican a los pobladores de estas áreas.

Las limitaciones y restricciones en el aprovechamiento sustentable de los recursos naturales que marca la ley, han sido la causa que la población local, en particular la mujer, emigre de sus comunidades en busca de nuevas experiencias laborales para sostener la economía familiar. Analizada de esta manera, se demuestra que las ANP cumplen de manera insatisfactoria con los objetivos básicos del interés público: el beneficio social, la distribución equitativa de la riqueza por género, el cuidado de los recursos naturales y la mejora de condiciones de vida de las personas. Más bien, parecen efectivos instrumentos administrativos para generar condiciones de desigualdad de género (Riemann et al, 2011)

En este sentido Lara (2008) plantea que el gran reto de la política ambiental está en desarrollar programas que aseguren la equidad entre los géneros, considerando como las relaciones de poder se reproducen jerárquica y asimétricamente entre hombre y mujeres, donde estas últimas son más afectadas en el acceso, manejo y control de los recursos forestales - como se advierte más adelante en las entrevistas realizadas para este estudio.

\section{Desigualdad de género: los intereses institucionales}

\section{y las realidades locales}

De acuerdo al Instituto Nacional de las Mujeres (INMUJ), la incorporación de la perspectiva de género en las políticas públicas que emanan del Poder Ejecutivo federal, ha constituido uno de los principales retos de la administración pública en México (INMUJ 2002). El informe oficial destaca la incorporación del discurso y la representación a las políticas institucionales, relevando avances significativos en la elaboración de planes y proyectos con perspectiva de género. El documento aclara sin embargo que, en el plano teórico, el género no solo se relaciona con las mujeres, por lo que en ningún momento el enfoque se centra en cuestionar los roles tradicionales femeninos.

Con la comprensión de que en la mayoría de las ANP en todo el mundo existen poblaciones que viven en su interior o en zonas adyacentes, las poblaciones viven sujetas a políticas y programas gubernamentales relacionados con los distintos tipos de actividades que las oficinas gubernamentales desarrollan en las áreas. Por ello, el Capítulo 24 de la Agenda 21, aprobada el 13 de junio de 1992 por 178 países en la Conferencia de las Naciones Unidas sobre el Medio Ambiente y el Desarrollo (Cumbre de la Tierra) de Río de Janeiro, donde plantea medidas que se deben tomar a nivel mundial, nacional y local en todos los ámbitos en los que los seres humanos afectan directamente la tierra, en la sección II-24 incluye la participación de las mujeres para lograr este desarrollo (ONU, 1992). Por su parte, la Convención para la Lucha contra la Desertificación de las Naciones Unidas (UNCCD por sus siglas en inglés) cuyo acuerdo Internacional universal busca promover una respuesta global para la desertificación, que entró en vigencia el 26 de diciembre de 1996, estipula una serie de medidas para promover la participación efectiva de las mujeres en la planificación de políticas, la adopción de decisiones, la ejecución y revisión de los programas nacionales de lucha contra la desertificación. Lo que se trata de explicar es que efectivamente existen, aunque sea en el discurso, aproximaciones pero no suficientes a la incorporación de las mujeres para ser sujetos de participación en el desarrollo de las ANP.

Por otro lado, la etnografía como metodología cualitativa expuesta por Geertz (1991, Aguilar, Castañeda y Salazar 2002) permite observar la necesidad de promover el capital social e incorporar la perspectiva de género en las políticas públicas ambientales en ANP, realidad que evidencia que existe un amplio discurso relacionado con el desarrollo sostenible, más que un conocimiento real de las demandas específicas de las poblaciones locales, en particular de las mujeres, quienes se sitúan solo como espectadoras de los proyectos productivos sustentables que por ley allí se implementan, elaborados siempre desde la institucionalidad con pensamiento masculino, acción que sigue fomentando y extendiendo en esas zonas un amplio margen en desigualdad de género.

En este sentido, y abonando a la discusión del papel de las mujeres en las áreas naturales protegidas, es necesario indagar por qué el diseño de las políticas públicas se orientan más al sexo masculino, discriminando el papel de las mujeres en el desarrollo de estas áreas. Podría pensarse por un lado, que quienes diseñan y deciden son hombres que amparados en una normatividad deciden los planes y programas de manejo ambientalmente sustentables donde la responsabilidad de implementación de los mismos es adjudicado a los hombres por su fuerza, o una segunda razón es la idea errónea de lo que significa la visión de género y la asociación de esta palabra con debilidad, pese a los grandes aportes que las mujeres han generado al desarrollo, como lo expone Vizcarra (2008) y a los desarrollos de la teoría de género; las desigualdades sociales que afectan 
directamente la vida de las mujeres, no sólo siguen reproduciéndose sino que se complejizan colocándolas en situaciones de mayor desventaja, marginación y pobreza.

En este sentido y como lo plantea Vizcarra (2008) siguiendo en esto a la Organización de las Naciones Unidas para la Alimentación y la Agricultura (FAO 1996), se le debe dar valor a los conocimientos especializados de las mujeres en relación con los recursos genéticos aplicados a la agricultura y la alimentación, que hacen de ellas custodias esenciales de la diversidad biológica. Por tal razón la misma invita a las instituciones a reconocer la utilidad de aprender de los conocimientos locales de las mujeres con miras a proteger y mantener el medio ambiente, tal y como lo recomienda la Agenda 21 en su apartado de Mujer en el desarrollo sustentable, pues el agua, el bosque, la tierra y la biodiversidad están presentes en muchos niveles de la vida de las campesinas, en este sentido es una cualidad de mucho valor de las mujeres que no se reconocen en el diseño de las políticas públicas para las zonas ambientalmente protegidas (ONU,1992).

\section{Zona de estudio: dos políticas públicas en contradicción}

La Reserva de la Biosfera del Alto Golfo de California y Delta del Río Colorado (RBAGDRC) es ampliamente reconocida por su relevancia ambiental, histórica y cultural a nivel internacional. Este reconocimiento como zona de gran biodiversidad sustentó el criterio para que el 10 de junio de 1993 fuera decretada oficialmente como área natural protegida, con una superficie de 934,756-25-00 ha, integrada por aguas de jurisdicción federal del Golfo de California y terrenos de los municipios de Mexicali (estado de Baja California) y San Luis, Río Colorado y Puerto Peñasco (estado de Sonora) (SEMARNAT, 2007:14-15)

A pesar que en México existe una legislación ambiental que atiende las ANP, el sentido que expresa la política fundamentada en el criterio de atención al territorio como reserva de la biosfera es el de conservación, condición que ha generado grandes cambios en las actitudes y percepciones con respecto al suelo que se ha configurado a través del tiempo y de las culturas. La legislación que ampara la reserva de la biosfera estipula que en la formulación y la aprobación del plan de manejo deben participar las comunidades que existen en ese ámbito. En el caso de la RBAGDRC, los principales usuarios del suelo y los recursos son, además de los pescadores comerciales, pesca deportiva, los propietarios turísticos, los agricultores, los organismos no gubernamentales y la población local (SEMARNAT, 2007).

Los actuales administradores de la reserva están enfrentando cambios de adaptación del conocimiento local tradicional de los ejidatarios y sus formas de aproximarse hacia un proceso de desarrollo sustentable. Sin embargo, la incorporación de la mujer en los proyectos productivos sustentables se ve minimizada en la zona, porque esos proyectos (forestación, ecoturismo y restauración de surcos) se enfocan hacia los hombres por requerir de esfuerzo fisico, condición que reafirma la visión errónea de que la mujer es débil y frágil (Entrevista al Director de la Comisión Nacional de Áreas Naturales Protegidas (CONANP) de San Luis Rio Colorado, realizada el 20 de octubre de 2014).

Los criterios institucionales para seleccionar los provectos productivos sustentables en la RBAGDRC, incluyen preservación de la diversidad biológica y de las costumbres y los estilos de vida de los residentes del área, atención a los principios de sustentabilidad, no interferencia en la aplicación de otro proyecto productivo en la zona, no ser excluyentes en diferentes localidades -debido a los diferentes temas que se pueden atender dentro de la reserva los proyectos productivos que se seleccionen no deben centrarse en una sola área, debiendo buscar la diversidad- y, finalmente, que no sean contingentes -es decir que el proyecto seleccionado no debe atender o dar respuesta a una catástrofe ambiental (SEMARNAT, 2007). Aunque parciales, estos criterios proporcionan un marco para advertir que en ningún momento se habla de género, o sea que la inclusión de las mujeres como agentes activos en los proyectos no figura ni en el texto ni en el discurso de las autoridades de la zona.

"En nuestra área de influencia existen dos condiciones muy específicas para seleccionar un proyecto: 1) que exista disponibilidad de financiamiento $y, 2)$ que la mano de obra esté disponible, preferentemente hombres, por el tipo de trabajo rudo que requiere el plan" (Entrevista, Director de la CONANP San Luis Rio Colorado, 20 de octubre de 2014)

Desde la perspectiva de Murrieta (2003), estas dos condiciones son atendidas por la política mexicana de conservación, donde existen algunos soportes financieros para hacer frente a los cambios de conservación y participación comunitaria en la protección de áreas, o al menos para mantener la administración del sitio, situación que hasta el momento parece no haber sido concretada en la zona, al presentar una contradicción de enfoques e intereses entre administradores y ejidatarias, pero sobre todo exponer cómo la desigualdad de género se construye a pesar de estar contemplado en la visión de la política ambiental.

Para lograr una aproximación a la comprensión de la desigualdad de género en las ANP, se debe reconocer que existen dos políticas públicas en permanente contradicción en un mismo territorio: una de conservación y otra de desarrollo humano, fenómeno que tiene origen en un proceso mal planeado y desarticulado que el gobierno federal implementó en la década de 1970 como estrategia para aminorar la presión social y demográfica y que hasta la fecha sigue vigente, generando una constante disputa entre administradores de la reserva y los ejidatarios de la zona, en particular de las mujeres, quienes se quejan de la falta de oportunidades de acciones locales para su economía familiar.

En este contexto de disputa constante, no siempre existe coincidencia entre dos actores tan desiguales en un mismo territorio, argumento que se sustenta en que sus propósitos 
e intereses son diferentes. Con esto se desea explicar que no solamente los intereses son disímiles, sino que el canal de diálogo entre los administradores y las ejidatarias, no cumple con su objetivo primordial de informar los planes de desarrollo a la población local. Situación que se constata en el Ejido Luis Encinas Johnson.

\section{Ejido Luis Encinas Johnson: la realidad desigual de las mujeres}

El Ejido Luis Encinas Johnson se encuentra localizado dentro del municipio de San Luis Río Colorado Sonora, en la parte sureste del Valle Agrícola de Mexicali (Gráfico). Este ejido tiene una superficie total de 6,092 hectáreas, sin incluir el núcleo poblacional de 25 hectáreas que se encuentra a 3 kilómetros al norte el polígono del Ejido y está ubicado entre los Ejidos Mesa Rica y Roberto de la Madrid.

El Ejido limita hacia el norte con el Ejido Mesa Rica, hacia el noreste con el Ejido Flor del Desierto, al noroeste con el Ejido Roberto de la Madrid, hacia el suroeste con el Ejido Francisco Aguilar Espinoza, y hacia el sur con la Reserva de la Biosfera del Alto Golfo de California y Delta del Río Colorado.

\section{Grafico 1.}

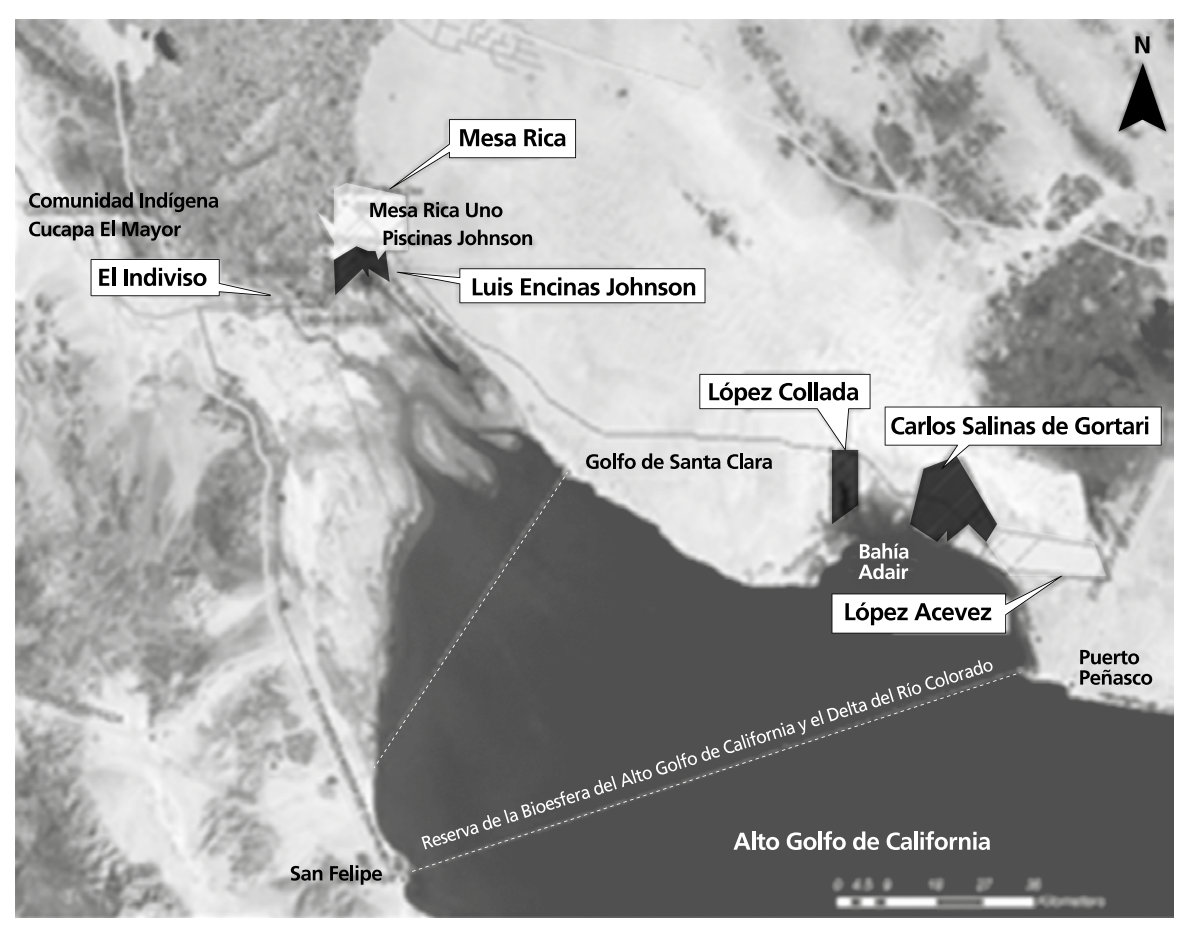

Fuente: PRONATURA, 2014.
El Ejido cuenta con 44 ejidatarios de los cuales el 67\% son hombres y 33\% mujeres Es una población rural con alto grado de marginación social y económica; de acuerdo a la Cruzada Nacional contra el Hambre la mayoría de los ejidatarios se encuentran viviendo fuera del ejido por motivos laborales (SEDESOL 2014). ${ }^{3}$ Con relación a su representatividad-organizativa, los recursos se gestionan para tratar los problemas y necesidades comunitarias a través de la mesa directiva. La autoridad máxima es la asamblea ejidal en la que participa el total de los ejidatarios. La asamblea sesiona cada mes por lo menos, para discutir los asuntos que conciernen a su población, su objetivo es mantener una eficiente administración de los recursos (económicos y naturales) con los que cuenta el Ejido. Se trata de llegar a un consenso por mayoría de votos, en base a esto la mesa directiva toma la decisión.

En este proceso de gestión y a pesar de las buenas intenciones que presentan los administradores de la reserva con respecto a los proyectos productivos, la realidad muestra que existen restricciones para generar alternativas de desarrollo local, bajo el argumento de que los planes propuestos, o no son sustentables, o simplemente no hay financiamiento. Según datos identificados por la Comisión Nacional de Áreas Naturales Protegidas (CONANP) y la organización ambiental PRONATURA, "las propuestas no emergen de la comunidad, lo que deja a los administradores tomar la decisión, dejando de lado la oportunidad de que las mujeres puedan incidir en la generación de opciones económicas que contribuyan a la mejora de su calidad de vida" (Rivas, Entrevista, 2014).

Por estas razones se explica que existen pocos datos acerca de la evolución económica del ejido, pero según refieren las ejidatarias en las entrevistas realizadas, este fenómeno de desigualdad de género está asociado a la ausencia de oportunidades laborales, incorporación en procesos de consulta sobre el desarrollo local de la zona y las condiciones mínimas de infraestructura social, responsabilidad de las dependencias federales en materia de conservación y preservación de los ecosistemas locales. En conjunto, todo parece indicar que la idea se enfoca a condenar al olvido a todos los ejidos.

Por los argumentos antes vertidos, se desprende que las políticas de conservación no han logrado armonizar con el desarrollo humano sin importar el género. Se evidencia claramente la ausencia de una voluntad política de las dependencias ambientales de promover un desarrollo local que incorpore a la mujer en los proyectos productivos sustentables. Así lo dejan entrever los siguientes testimonios

"desde antes de la declaración habían prometido invertir en la zona de parte del gobierno federal [...] las ejidatarias necesitamos programas y proyectos que nos generen ingresos para comer y mantener a la familia [...] al no cumplir las promesas tenemos que emigrar en busca de 
otras alternativas económicas...” (Entrevista, María, 44 años, ejidataria por 10 años, 2014).

"Desde siempre solo los hombres pueden trabajar en los proyectos de forestación o limpia de terrenos, no tengo recuerdos que se hayan acercado para invitarme a trabajar [...] ni mucho menos para decirme la razón por la cual nos excluyen. A mi entender solo nos dan atole con el dedo, hasta hoy no comprendo sus intenciones de desarrollo en esta zona [...] generalmente, siempre hemos percibido que nos quieren fuera del área [...] al fin $y$ al cabo parcialmente ya lo lograron porque trabajamos en el Golfo de Santa Clara, pero sí creo que las mujeres tenemos mínimas oportunidades de trabajar aqui"' (Martina, 62 años, ejidataria por 32 años, 2014).

Como resultado de la falta de políticas y recursos para que la población local pueda realizar proyectos productivos sustentables para su manutención, el desarrollo de la zona queda prácticamente en manos de los administradores, situación que no es bien observada por las ejidatarias cuando refieren que

"nunca hay dinero para nada, solo que si podemos ayudar sin pago [...] para eso no importa que seamos mujeres, al fin $y$ al cabo nos representan los hombres [...] lo cierto es que nosotras ni siquiera existimos en la zona; todo lo comentan, informan o negocian con los hombres, quienes en ocasiones nos dicen algo en la junta ejidal" (Teresa, 66 años, ejidataria por 38 años, 2014).

"Para los administradores, la justificación de rechazo a las mujeres en las propuestas productivas, tiene una relación directa con las condiciones adversas del entorno físico natural, el costo-beneficio de los proyectos y el ambiente de austeridad de financiamiento en que se desarrollan las áreas naturales protegidas del mundo, y especificamente, la RBAGDRC" (Entrevista Director de la CONANP San Luis Rio Colorado, realizada el 20 de octubre de 2014).

Esta percepción de los administrativos con respecto al escenario actual en materia de proyectos productivos sustentables, se puede observar en el siguiente comentario: "la función de la administración de la reserva es recoger y analizar las demandas de los ejidatarios, con la finalidad de consolidar el binomio conservación-aprovechamiento de los recursos naturales, tarea por demás nada fácil, cuando los proyectos comunitarios impliquen un elevado incremento de recursos financieros, que en una etapa de recorte presupuestal y fase de austeridad, se refleja en el rechazo de la mayoría de propuestas locales sin importar si son hombres o mujeres" (Entrevista Director de la CONANP San Luis Rio Colorado, realizada el 20 de octubre de 2014).

Los datos presentados revelan dos situaciones: primero que las políticas públicas aplicadas en la RBAGDRC desde la óptica de los administradores, están enfocadas hacia la conservación de los recursos naturales de la zona, pero con alto grado de marginación al acceso de las mujeres en las propuestas productivas sustentables, con intención o por omisión; y dos que los grados mínimos de desarrollo humano en los ejidos situados en la zona, en particular en Luis Encinas Johnson, muestran cómo la desigualdad de género ha estado presente desde la declaratoria de la zona como Área Natural Protegida. Ello nos lleva a reconocer que efectivamente existen dos políticas públicas en contradicción: una de conservación y otra de desarrollo humano.

\section{A modo de conclusión}

Para fines de este trabajo, el tema de la desigualdad de género se comprende como el desigual acceso que por razones culturales tienen las personas según el sexo a los recursos económicos y sociales, destacando que este fenómeno es visible y común en las áreas naturales protegidas de México. Pero el mayor reto de confrontar la economía con la política ambiental es entender en qué nivel de participación del desarrollo local se encuentran las comunidades insertas en las Áreas Naturales Protegidas, así como reconocer si las demandas sociales corresponden a la práctica discursiva de las dependencias y organismos nacionales e internacionales que justifican el aprovechamiento racional sustentable de los recursos naturales en estas zonas, un ejemplo de ello es la Agenda 21 donde en uno de sus apartados invita a los responsables de diseñar estos programas en áreas protegidas a incluir a las mujeres dada la capacidad que estas históricamente han demostrado en la conservación y desarrollo del medio ambiente.

La contradicción de dos políticas públicas en un solo territorio: una de conservación y otra de desarrollo humano, ha generado un escenario de desigualdad de género directa e indirectamente, tomando en cuenta que negar las oportunidades de lograr mejores condiciones de vida a las mujeres a través del uso responsable de los recursos naturales 
en su territorio, no solo las discrimina y dificulta su visibilidad, sino que las restringe como ciudadanas y sujetos beneficiarios del bien público.

La desigualdad de género es entendida en las políticas públicas como una circunstancia que ha impulsado a un segmento de la población local del Ejido Luis Encinas Johnson a emigrar y emplear su mano de obra fuera de la localidad, en particular las mujeres al no ser tomadas en cuenta para incorporarse en proyectos productivos sustentables; en ese sentido, el proceso de desarraigo de las mujeres respecto a su comunidad supone una percepción de despojo de los recursos aprovechados tradicionalmente (Riemann et al, 2011).

Desde una perspectiva amplia del desarrollo local, se considera que falta mucho para entender que mientras las estructuras políticas y las actitudes culturales no adopten una perspectiva de género en políticas ambientales, la incorporación de la mujer en programas y planes en la zona estará solo en discurso, a la vez que continúe persistiendo la visión de los administradores donde prevalezca el control social de orden patriarcal. Ante esta situación, es imperante exigir a la administración pública que cumpla con los acuerdos internacionales de incorporar a la mujer en los programas de política ambiental; ya no existe excusa válida que justifique la desigualdad de género en algún ámbito, y mucho menos en estas áreas, territorios que deben convertirse en espacios de oportunidad para el desarrollo sostenible, que incrementen la calidad de vida de los pobladores, principalmente el de las mujeres y abatir la contradicción entre las políticas de desarrollo aquí analizadas.
El ejido es una sociedad de interés social, integrada por campesinos mexicanos por nacimiento, con un patrimonio inicial u originario constituido por las tierras, bosques y aguas que el Estado les entrega gratuitamente en propiedad inalienable, intransferible, inembargable e imprescriptible; sujeto a su aprovechamiento y explotación a las modalidades establecidas en la ley, bajo la orientación del estado en cuanto a la organización de su administración interna, basada en la cooperacion y la democracia economica y que tiene por objeto la explotación y el aprovechamiento integral de sus recursos naturales y humanos, nediante el trabajo persona de sus socios (ejidatarios) en su propio beneficio (Ruiz Massieu, 1990)

${ }^{2}$ Participaron el Director de la Comisión Nacional de Áreas Naturales Protegidas (CONANP) encargado de disenarar y llevar a cabo los proyectos de conservación ambiental, y cuatro mujeres ejidatarias que expresan que accedieron a participar en la entrevista lo hicieron de manera informada y voluntari que las persona empleada es cualitativa. Las mujeres entrevistadas son cabeza de familia que habitan en el ejido Luis Encinas Johnson desde hace más de diez años.

${ }^{3}$ La Cruzada Nacional contra el Hambre es una estrategia de política social, integral y participativa del gobierno mexicano, que pretende una solución estructural y permanente a un grave problema que existe en el país: el hambre, posee un enfoque de caracter integral que involucra mültiples instrumentos de politica publica en materia de alimentación, salud, educación, vivienda, servicios en la vivienda e ingresos (SEDESOL, 2013). 


\section{Referencias}

Aguilar Villanueva, Luis F. (2007) "Estudio introductorio" En La hechura de las políticas públicas. Colección de Antologías de Políticas Públicas. México: Miguel Ángel Porrúa, págs. 15-83.

Aguilar, Lorena; Itzá Castañeda e Hilda Salazar (2002). En busca del género perdido. San José, Costa Rica: Editorial Absoluto S.A.

Bourdieu, Pierre (2007) La dominación masculina. Barcelona: Editorial Anagrama.

Comisión Nacional de Áreas Naturales Protegidas (2009) Programa Nacional de Áreas Naturales Protegidas. México: CONANP.

FAO, Organización de las Naciones Unidas para la Alimentación y la Agricultura (1996) El estado mundial de la agricultura y la alimentación. Roma: Fao.

Ferrajoli, Luigi y Miguel Carbonell (2005) Igualdad y diferencia de género. México: Colección Miradas 2.

Instituto Nacional de las Mujeres (2002) Perfil de mujeres y hombres en México. México: INMUJ.

Lamas, Martha (1986) "La antropología feminista y la categoría de género". En: El género, la construcción cultural de la diferencia sexual. Ciudad de México: PUEG/UNAM, págs.92-126.

Lara, A. Silvana, B. Ivonne Vizcarra, (2008) "Políticas ambientales-forestales y capital social femenino Mazahua”. Economía, Sociedad y Territorio, 26: DOI: http://dx.doi org/10.22136/est002008214

Maffia, Diana (2006) "Lo que no tiene nombre" En Disidencia sexual e identidades sexuales y genéricas. Ciudad de México: Consejo Nacional para Prevenir la Discriminación (CONAPRED), págs.31-35.

Mejía S. Carlos y Pamela Henríquez R. (2012) "La ciudadanía como co-construcción de espacios de participación en lo público". Sociología (Porto Alegre), 31:192-213.

ONU, Organización de las Naciones Unidas (1992) Conferencia de Naciones Unidas sobre el Medio Ambiente y el Desarrollo (UNCED). Agenda 21, cap. 24. Río de Janeiro, Brasil. Disponible en: http://www.un.org/spanish/esa/sustdev/agenda21/agenda21spchapter24.htm [25 de junio de 2017].
Oliveira, Orlandina de (2007) "Reflexiones acerca de las desigualdades sociales y el género". Estudios Sociológicos, 75: 805-812.

PNUD, Programa de las Naciones Unidas para el Desarrollo (2013) Informe del Desarrollo Humano: El ascenso del Sur. Madrid: PNUD.

PNUMA, Programa de las Naciones Unidas para el Medio Ambiente (2007) Informe Anual. Madrid: PNUMA.

Postigo A. Martha (2006) Género e igualdad de oportunidades: la teoría feminista y sus implicaciones éticas-políticas. Málaga: Universidad de Málaga.

Rawls, John (1997). Teoría de la justicia. Ciudad de México: Fondo de Cultura Económica.

Riemann, Hugo; Ricardo V. Santes-Álvarez y Alberto Pombo (2011) "El papel de las áreas naturales protegidas en el desarrollo local. El caso de la península de Baja California”. Gestión y Política Pública. México, 'págs.141-172.

Ruiz Carbonell, R. (2009) El principio de igualdad entre hombres y mujeres. Del ámbito público al ámbito jurídico familiar. Barcelona: Universidad de Barcelona.

Ruiz Massieu, M. (1990) Derecho Agrario. Universidad Nacional Autónoma de México Disponible en: https://archivos.juridicas.unam.mx/www/bjv/libros/2/913/11.pdf] [25 de junio de 2017].

SEDESOL, Secretaría de Desarrollo Social (2013). Cruzada Nacional contra el hambre. México. Disponible en: https://www.gob.mx/sedesol/acciones-y-programas/cruzada-nacional-contra-el-hambre-18938 [15 de junio de 2017].

SEDESOL, Secretaría de Desarrollo Social (2014) Elementos técnicos de diseño, planeación e instrumentación del programa nacional México sin Hambre. http://www.sedesol. gob.mx/work/models/sedesol/Programa/Cruzada_Nacional_Meexico_Sin_Hambre Elementos_Metodologicos.pdf (16 de junio 2017).

SEMARNAT, Secretaría del Medio Ambiente y Recursos Naturales (2007) Programa de Conservación y Manejo Reserva de la Biosfera Alto Golfo de California. México: SEMARNAT-CONANP

Sen, Amartya (1993) "Capability and Well-being", en M. Nussbaum y A. Sen (eds.) The Quality of Life. Oxford, Clarendon Press. Tercera reimpresión, México, Fondo de Cultura Económica, 2002) 
Soto Romero, G. (2013) El Estado como garante de los derechos fundamentales de las mujeres en Venezuela bajo el marco de la nueva ley orgánica de los derechos de las mujeres a una vida libre de violencia. Madrid: Universidad Nacional de Educación a Distancia.

Subirats, Joan y Gomá, Ricard (2000) "Cambios en las políticas de bienestar e innovación social en la Unión Europea”. Reforma y Democracia, 17: 47-76.

Varela, G. Helena M. (2012) "Iguales, pero no tanto. El acceso limitado de las mujeres a la esfera pública de México”. CONfines, 16:39-67.

Velázquez G. Margarita (2005) "El monte se va a acabar: las percepciones de hombres y mujeres acerca del futuro de la selva en la zona maya de Quintana Roo", en Paola Seisa y Emma Zapata (coords.), Transformaciones del campo mexicano: una mirada desde los estudios de género. Ciudad de México: AMER-CONACYT-Gobierno de ZacatecasPraxis, México, pp. 69-112.

Viola, Andreu (2000). Antropología del desarrollo. Teorías y Estudios Etnográficos en América Latina. España: Paidós.

Vizcarra B., Ivonne (2008) "Entre las desigualdades de género: un lugar para las mujeres pobres en la seguridad alimentaria y el combate al hambre". Argumentos, 57:141-170 Universidad Autónoma Metropolitana Unidad Xochimilco.

Warman, Arturo (2001) El campo mexicano en el siglo XX. México: Fondo de Cultura Económica.

Cómo citar este artículo

Jaramillo Cardona, Martha Cecilia y Salvador Adamé Gómez (2017) “Desigualdad de género y políticas públicas en áreas naturales protegidas". Revista Perspectivas de Políticas Públicas vol.7 № 13

Reseñ

\section{Emmanuel Biset y Roque Farrán (compiladores)}

\section{Estado: Perspectivas Posfundacionales}

Primera edición. Ciudad Autónoma de Buenos Aires. Prometeo Libros, 2017. 280 p. ISBN 978-987-574-843-9.

Sebastián Cruz Barbosa Universidad Nacional de Lanús . 